\title{
Estudio Palinológico de los helechos y licofitas de la Zona Núcleo Poniente de la Reserva Ecológica del Pedregal de San Ángel, Ciudad de México, México \\ PALYNOLOGICAL STUDY OF THE FERNS AND LYCOPHYTES OF THE CORE WEST ZONE OF the Ecological Reserve of the Pedregal of San Angel, Mexico City, Mexico
}

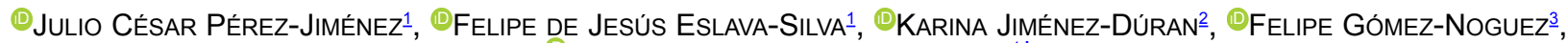

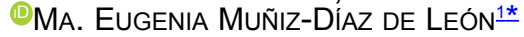

${ }^{1}$ Facultad de Ciencias, Universidad Nacional Autónoma de México, Ciudad de México, México.

${ }^{2}$ Facultad de Química, Universidad Nacional Autónoma de México, Ciudad de México, México.

${ }^{3}$ Herbario de la Facultad de Ciencias Químico Biológicas, Universidad Autónoma de Guerrero, Guerrero, México.

*Autor de correspondencia: mathe18@yahoo.com.mx

\section{Resumen}

Introducción: La Reserva Ecológica del Pedregal de San Ángel (REPSA) corresponde a un matorral xerófilo. En ella, los helechos y licofitas crecen en diversos microhábitats. En esta zona se han realizado investigaciones ecológicas y taxonómicas de la vegetación donde se han incluido las pteridofitas, sin embargo, no existen estudios palinológicos para este grupo de plantas.

Pregunta: ¿Cuál es la micromorfología de las esporas de las especies de helechos y licofitas de la REPSA?

Especies bajo estudio: Helechos y licofitas.

Sitio de estudio: Zona Núcleo Poniente (ZNP) de la Reserva Ecológica del Pedregal de San Ángel, Ciudad Universitaria, Ciudad de México, México. Agosto 2013 a septiembre 2016.

Métodos: Se recolectaron en campo ejemplares de referencia de helechos y licofitas, se identificaron y se depositaron en el Herbario Nacional (MEXU). Se obtuvieron las esporas a partir de pinnas fértiles, se montaron para observarse y medirse en microscopía fotónica (MF) y microscopía electrónica de barrido (MEB). Se obtuvieron las microfotografías y se realizó la descripción palinológica.

Resultados: Se presenta la palinología de 23 especies de helechos y dos de licofitas, correspondientes a 16 géneros. Se describe por primera vez la micromorfología de las esporas de: Gaga decomposita, G. membranacea, Myriopteris allosuroides, Pellaea cordifolia, Pellaea villosa, Pleopeltis thyssanolepis, Ophioglossum engelmannii y Selaginella sartorii. Para las restantes especies se complementa y/o corrobora la micromorfología.

Conclusiones: Los datos aportados contribuyen al conocimiento palinológico de las especies de licofitas y helechos presentes en la REPSA, y son caracteres de gran utilidad para la taxonomía de las especies.

Palabras clave: Esporas, helechos, licofitas, micromorfología, ornamentación.

\begin{abstract}
Background: The Pedregal of San Angel Ecological Reserve (REPSA) constitutes a xerophilous scrub. In the Reserve, both ferns and lycophytes (pteridophytes) grow in different microhabitats. Ecological and taxonomic studies of the vegetation where pteridophytes have been included have been carried out in this area. However, there are no palynological studies from this group of plants in the REPSA.

Question: ¿What is the spore micromorphology of the fern and lycophyte species from the REPSA?

Studied species: Ferns and lycophytes

Study site: West Core Zone (ZNP) in The Pedregal of San Ángel Ecological Reserve, Ciudad Universitaria, (South of Mexico City) Mexico City, Mexico. August 2013 to September 2016.

Methods: Reference specimens from ferns and lycophytes were collected, identified, and deposited at the National Herbarium (MEXU). Spores were recovered from fertile pinnae and mounted for photonic microscopy and scanning electron microscopy. Microscopy images were captured to carry out the palynological description.

Results: The palynology of 23 species of ferns and two species of lycophytes, corresponding to 16 genera, is presented. The spore micromorphology of Gaga decomposita, G. membranacea, Myriopteris allosuroides, Pellaea cordifolia, Pellaea villosa, Pleopeltis thyssanolepis, Ophioglossum engelmannii and Selaginella sartorii, is described here for the first time. Micromorphology is supplemented and / or confirmed for the remaining species.

Conclusions: Data provided in this work contribute to the palynological knowledge of the species of lycophytes and ferns present in the REPSA and are useful characters for the taxonomy of species.
\end{abstract}

Key words: Ferns, lycophytes, micromorphology, ornamentation, spores.

Este artículo se encuentra bajo los términos de la licencia Creative Commons Attribution License CCBY-NC (4.0) internacional. 
Los helechos y licofitas se encuentran adscritos a las clases Polypodiopsida y Lycopodiopsida respectivamente, de acuerdo con PPGI (2016). Las características morfológicas del esporofito han sido importantes herramientas para delimitar jerarquías taxonómicas, principalmente a nivel específico. Las esporas son estructuras de resistencia haploides que presentan caracteres micromorfológicos como son el tamaño, la forma y la ornamentación, los cuales se han utilizado para delimitar a las especies de helechos y licofitas (Tryon \& Tryon 1982, Lellinger \& Taylor 1997, Contreras-Duarte et al. 2006). Existen diversos estudios que abordan el campo de la palinología de los helechos y licofitas, siendo el más representativo para el grupo el de Tryon \& Lugardon (1991). Muchos trabajos palinológicos han sido de gran utilidad para la delimitación de familias, géneros y especies (Kremp \& Kawasaki 1972, Tryon \& Lugardon 1991) por ejemplo: Cystopteris (Hagenah 1961), Thelypteridaceae (Wood 1973), Cheilanthes (Knobloch 1969, Knobloch et al. 1971), Notholaena (Devi et al. 1971), Anemia (Hill 1977, 1979) y Serpocaulon (Ramírez-Valencia et al. 2013). Distintos autores han elaborado descripciones detalladas de las esporas de distintos grupos de helechos y licofitas, lo que ha servido en la reconstrucción filogenética de los mismos, como es el caso de Cheilanthes (Knobloch et al. 1971), Cyatheaceae (Gastony 1979), Polypodium (Lloyd 1981), Marsileaceae (Schneider \& Pryer 2002), Selaginellaceae (Korall \& Taylor 2006), Elaphoglossum (Moran et al. 2007), Lomariopsis (Rouhan et al. 2007), Leucothrichum (Labiak et al. 2010), Lindsaeaeceae (Lehtonen et al. 2010), Serpocaulon (Ramírez-Valencia \& Sanín 2017) y Pteris (Chao \& Huang 2018), entre otros.

En México se han realizado estudios palinológicos locales para helechos y licofitas. Entre ellos se destacan los realizados para Nuevo León (Arreguín-Sánchez \& AguirreClaverán 1990), el Valle de México (Arreguín-Sánchez 1985, Querétaro (Arreguín-Sánchez et al. 1996) y en Hidalgo (Gómez-Noguez et al. 2013). Sin embargo, es necesario continuar con el estudio de estas estructuras para seguir contribuyendo al conocimiento de la palinología en México.

El presente trabajo aborda el análisis de la micromorfología de las esporas de las especies de helechos y licofitas presentes en la Zona Núcleo Poniente (ZNP) de la Reserva Ecológica del Pedregal de San Ángel (REPSA). Se describen de forma detallada las esporas de 23 especies de helechos y dos de licofitas, correspondientes a 16 géneros y a ocho familias.

\section{Materiales y métodos}

Área de estudio. La REPSA se encuentra localizada al Suroeste de la Ciudad de México $19^{\circ} 18$ '31 "- $19^{\circ} 19$ '17"
Norte, $99^{\circ} 10$ '20 "- $99{ }^{\circ} 11$ ' 52 " Oeste, a 2,200-2,700 m snm, cuenta con una superficie total de 237.3 ha $\left(2.373 \mathrm{~km}^{2}\right)$. La ZNP abarca 94 ha $\left(0.94 \mathrm{~km}^{2}\right)$ y está limitada al Oriente por la Avenida de los Insurgentes, al Sur por la subestación eléctrica Ingeniero Odón de Buen, la Colonia Jardines del Pedregal de San Ángel (CJPSA), la Zona de Amortiguamiento Vivero Alto y los límites del Colegio de Ciencias y Humanidades Plantel Sur, al Poniente por los predios de CJPSA, y al Norte por el Instituto de Biología, la Zona de Amortiguamiento del Jardín Botánico y los Institutos de Ecología e Investigaciones Biomédicas (Figura 1). La REPSA representa un matorral xerófilo con vegetación de transición, con una marcada temporada de lluvias y períodos de sequías, la precipitación anual en el área es de $870.2 \mathrm{~mm}$ (Valiente-Banuet \& De Luna 1990, Cano-Santana et al. 2009).

Colecta e identificación de material biológico. Se realizaron doce colectas en la ZNP de la REPSA, seis correspondientes a la temporada de lluvias (julio-octubre) y seis en temporada de secas (noviembre-marzo), durante los años 2013-2016. La recolecta de los ejemplares de respaldo para cada especie de helechos y licofitas registrados, se realizó siguiendo la metodología de Lot \& Chiang (1986) y la obtención de las esporas, se efectuó de acuerdo con Muñiz-Díaz de León et al. (2008). Se colocaron frondas fértiles de las diferentes especies en sobres sellados de papel manila y se secaron durante una semana en una estufa a $35{ }^{\circ} \mathrm{C}$ a fin de liberar las esporas (MOD E-51, BG). Posteriormente el contenido de los sobres se pasó por un tamiz Mont Inox $\mathrm{n}^{\circ}$ 200, de $0.074 \mathrm{~mm}$ de diámetro para eliminar los residuos de pinnas y esporangios, las esporas se mantuvieron en viales a $4{ }^{\circ} \mathrm{C}$. Para la determinación de las especies se utilizaron las claves taxonómicas de Mickel \& Beitel (1988), Moran \& Riba (1995) y Mickel \& Smith (2004).

Montaje, observación y descripción de esporas. La observación de las esporas se realizó por medio de microscopía fotónica (MF) y microscopía electrónica de barrido (MEB). Para MF, se montaron y observaron 30 esporas por especie en campos al azar (excepto en las megasporas de Selaginella sartorii donde no se contaba con material suficiente), procedentes de diferentes individuos, y se evaluó la forma y contorno en vista polar (VP) y ecuatorial (VE), los diámetros ecuatorial y polar (valor mínimo, valor máximo y promedio \pm desviación estándar), así como la correlación entre ambos. El montaje de las muestras para MF se realizó de la siguiente manera: se tomaron las esporas con un pincel, se espolvorearon sobre un portaobjetos en una gota de resina sintética (Marca: Hysel), se cubrieron con un cubreobjetos y se secaron por dos semanas a temperatura ambiente. Se etiquetaron con el 


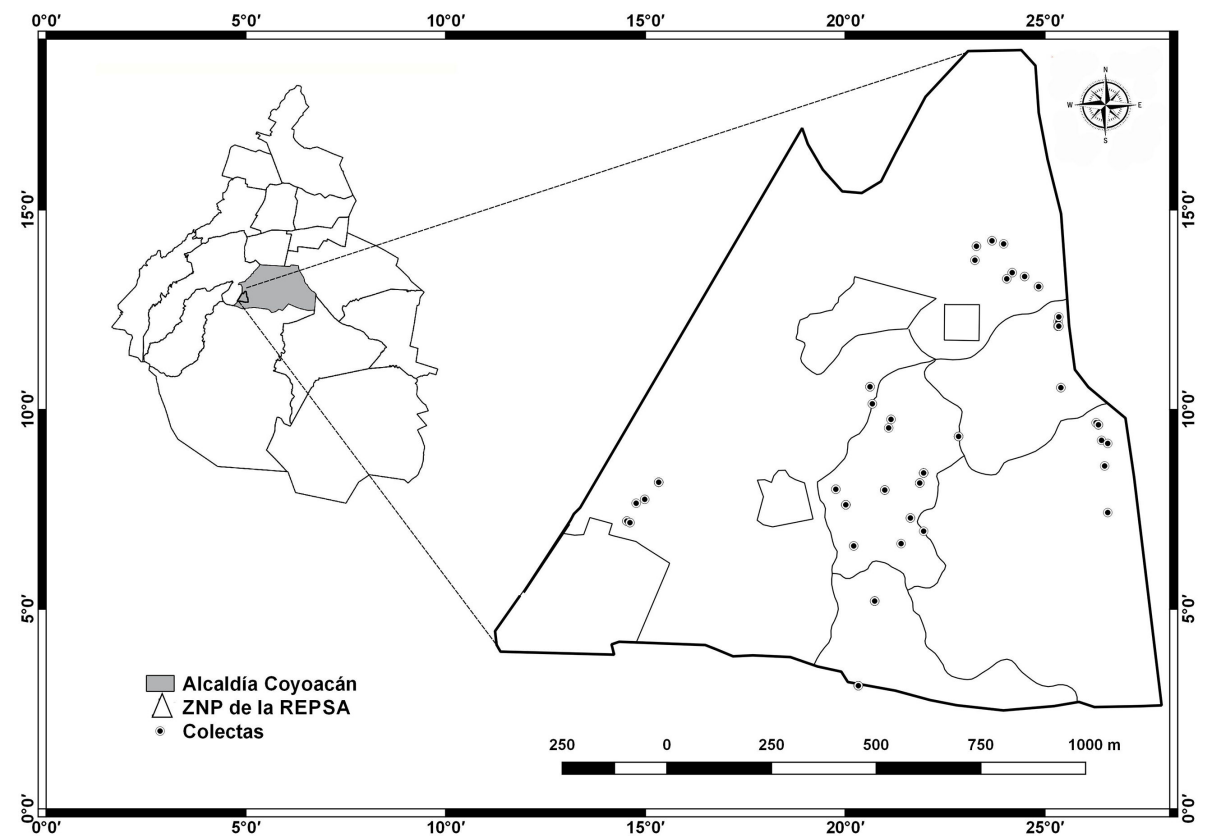

Figura 1. Localización del área de estudio, Zona Núcleo Poniente (ZNP) de la Reserva Ecológica del Pedregal de San Ángel (REPSA), Ciudad de México.

nombre de la especie y el número de colecta. La obtención de microfotografías de las esporas se realizó con un microscopio con cámara integrada marca Leica $^{\circledR}$ (Mod ICC50 E). Las medidas del eje polar y ecuatorial se obtuvieron con el programa LAS EZ que es parte de la paquetería con que cuenta el microscopio.

Para el análisis de la ornamentación (O) de las esporas se utilizó la técnica de MEB. Se tomaron esporas con un pincel y se espolvorearon sobre un porta muestras cubierto con cinta conductiva de carbón adhesiva de doble cara. Posteriormente se cubrieron con oro-paladio en espray (EMITECH k850) y se observaron al microscopio electrónico de barrido (Joel JSM-5900-LV). Se adquirieron las microfotografías de las esporas en vista polar, ecuatorial, distal, proximal, y se observaron los detalles de la ornamentación.

La descripción de las esporas de las diferentes especies se realizó de acuerdo a Kremp (1965), Nayar \& Devi (1966) y Punt et al. (2007) y para una correcta traducción se utilizó el glosario de Lellinger (2002).

\section{Resultados}

Se describió la micromorfología de las esporas de 23 especies de helechos y dos de licofitas, correspondientes a 16 géneros y a ocho familias de la ZNP de la REPSA. La familia con mayor representación fue Pteridaceae con 13 especies, seguida por Aspleniaceae y Polypodiaceae con tres especies cada una, Selaginellaceae con dos, y Cystopteridaceae, Dryopteridaceae, Ophioglossaceae y
Woodsiaceae con una sola especie cada una. Las esporas en Pteridaceae son triletes, la mayoría obladas esferoidales, sin embargo, también pueden ser proladas esferoidales y subobladas. La ornamentación que poseen es rugulada, cristada, psilada, reticulada o escábrida. Los miembros de Aspleniaceae presentan esporas monoletes, obladas o subobladas, la ornamentación es psilada o cristada. Por otro lado, las especies de Polypodiaceae son monoletes, obladas y la ornamentación es rugulada o psilada. Los diámetros y la forma de las esporas (relación eje polar/ecuatorial) se encuentran detallados en la Tabla 1.

Descripción micromorfológica de las especies. Aspleniaceae Newman. Asplenium fibrillosum Pringle \& Davenp. ex Davenp.- Espora monolete con perisporio evidente. VP: elipsoidal y margen irregular debido a los pliegues del perisporio. VE: elipsoidal, planoconvexa a reniforme, con contornos irregulares. O: reticulado en toda su superficie. Se aprecia el exosporio espinoso, cubierto por el perisporio con pliegues de hasta $5 \mu \mathrm{m}$ de alto, formando un patrón reticulado a lofado, en cuyas lagunas se pueden observar escasas microespinas y perforaciones. Se observan los pliegues del perisporio formando un retículo heterobrocado, con lumen de 5 a $14 \mu \mathrm{m}$ (Figura 2A-C y 8A). Ejemplar: Pérez-Jiménez 42, MEXU folio: 1465158. Previo a este estudio solo se conocía información acerca del diámetro (Dyer et al. 2012). El presente trabajo provee información acerca del tamaño del eje polar, eje ecuatorial, la forma y ornamentación de las esporas. 
Tabla 1. Diámetros y la forma de las esporas de los helechos y las licofitas de la ona Nuecleo Poniente de la Resvera del Pedregal de San Angel, Ciudad de Mexico.

\begin{tabular}{|c|c|c|c|}
\hline Taxón & Eje polar $(\mu \mathrm{m})$ & Eje ecuatorial $(\mu \mathrm{m})$ & $\begin{array}{c}\text { Forma: } \mathrm{P}: \mathrm{E}=\text { relación eje polar } \mathrm{y} \\
\text { ecuatorial }\end{array}$ \\
\hline Asplenium fibrillosum & $48.32 \pm 4.2(42.0-59.0)$ & $64.14 \pm 5.5(53.0-78.0)$ & 0.75 suboblado \\
\hline Asplenium monanthes & $26.74 \pm 1.9(25.0-30.0)$ & $42.55 \pm 6.8(25.0-50.0) \bullet$ & 0.62 oblado \\
\hline Asplenium praemorsum & $30.29 \pm 5.3(25.0-47.5)$ & $54.18 \pm 3.7(47.5-67.5) \bullet$ & 0.55 oblado \\
\hline Cystopteris fragilis & $25.35 \pm 2.3(22.5-37.5)$ & $38.80 \pm 3.2(30.0-47.5)$ & 0.65 oblado \\
\hline Dryopteris cinnamomea & $26.48 \pm 1.8(22.0-30.0)$ & $41.90 \pm 2.2(36.0-48.0)^{\bullet}$ & 0.63 oblado \\
\hline Ophioglossum engelmannii* & $36.7 \pm 2.1(31.9-43.6)$ & $35.6 \pm 2.0(31.5-39.7)$ & 1.03 prolado esferoidal \\
\hline Phlebodium pseudoaureum & $23.58 \pm 2.1(19.0-28.0)$ & $39.64 \pm 2.9(32.0-45.0)$ & 0.59 oblado \\
\hline Pleopeltis thyssanolepis* & $35.08 \pm 2.6(31.0-46.0)$ & $57.17 \pm 3.7(50.0-65.0)$ & 0.61 oblado \\
\hline Polypodium subpetiolatum & $31.94 \pm 3.2(25.0-44.0) /$ & $50.22 \pm 4.1(37.0-59.0) \bullet$ & 0.63 oblado \\
\hline Adiantum capillus-veneris & $43.24 \pm 2.9(27.5-47.5)$ & $44.17 \pm 1.9(40.0-48.0)$ & 0.97 oblado esferoidal \\
\hline Astrolepis sinuata & $46.4 \pm 3.6(35.8-52.9)$ & $59.0 \pm 3.4(50.7-64.8)$ & 0.78 suboblado \\
\hline Bommeria pedata & $48.32 \pm 4.2(52.5-58.0)$ & $64.14 \pm 5.5(53.0-78.0) \bullet$ & 1.14 prolado esferoidal \\
\hline Cheilanthes farinosa & $59.45 \pm 3.8(52.0-68.0)$ & $60.38 \pm 3.5(54.0-71.0)$ & 0.98 oblado esferoidal \\
\hline Gaga decomposita* & $67.60 \pm 5.0(58.0-78.0)$ & $66.62 \pm 5.0(55.0-78.0)$ & 1.01 prolado esferoidal \\
\hline Gaga kaulfussii & $61.82 \pm 4.0(46.0-68.0)$ & $63.50 \pm 4.4(46.0-72.0)$ & 0.97 oblado esferoidal \\
\hline Gaga membranacea* & $67.7 \pm 2.7(73.3-61.3)$ & $68.8 \pm 3.3(63.5-76.9)$ & 0.98 oblado esferoidal \\
\hline Myriopteris allosuroides* & $38.86 \pm 1.8(35.0-43.0)$ & $34.80 \pm 10.37(11.0-45.0)$ & 1.11 prolado esferoidal \\
\hline Myriopteris aurea & $60.59 \pm 3.0(55.0-68.0)$ & $61.24 \pm 3.9(53.0-72.0)$ & 0.98 oblado esferoidal \\
\hline Myriopteris myriophylla & $64.57 \pm 3.6(57.5-72.0)$ & $66.73 \pm 3.1(62.0-75.0)$ & 0.96 oblado esferoidal \\
\hline Pellaea cordifolia* & $45.53 \pm 3.4(40.0-59.0)$ & $46.25 \pm 2.4(42.0-51.0)$ & 0.98 oblado esferoidal \\
\hline Pellaea ternifolia & $48.70 \pm 3.9(37.5-56)$ & $50.50 \pm 3.6(42.5-57.0)$ & 0.96 oblado esferoidal \\
\hline Pellaea villosa* & $52.51 \pm 4.3(35.0-58.0)$ & $54.33 \pm 4.2(37.5-60.0)$ & 0.96 oblado esferoidal \\
\hline Selaginella pallescens microspora & $18.9 \pm 5.6(12.9-26.2)$ & $24.78 \pm 2.9(21.3-30.8) \bullet \bullet$ & 0.76 suboblado \\
\hline megaspora & $323.96 \pm 31.14(200.0-361.0)$ & $333.46 \pm 19.96(295.0-363.0)$ & 0.97 oblado esferoidal \\
\hline Selaginella sartorii microspora & $30.4 \pm 3.2(23.8-36.1)$ & $36.8 \pm 3.7(31.4-44.4)$ & 0.82 suboblado \\
\hline Woodsia mollis & $40.29 \pm 3.2(32.5-47.5)$ & $64.76 \pm 3.4(55.0-70.0) \bullet$ & 0.62 oblado \\
\hline
\end{tabular}

Eje ecuatorial y polar promedio \pm desviación estándar (valor mínimo - valor máximo)

*Descripción palinológica de esporas por primera vez.

- Las esporas descritas tienen diámetros mayores con respecto a estudios previos.

- Las esporas descritas tienen diámetros menores con respecto a estudios previos. 
Pérez-Jiménez et al. / Botanical Sciences 98(3): 517-532. 2020
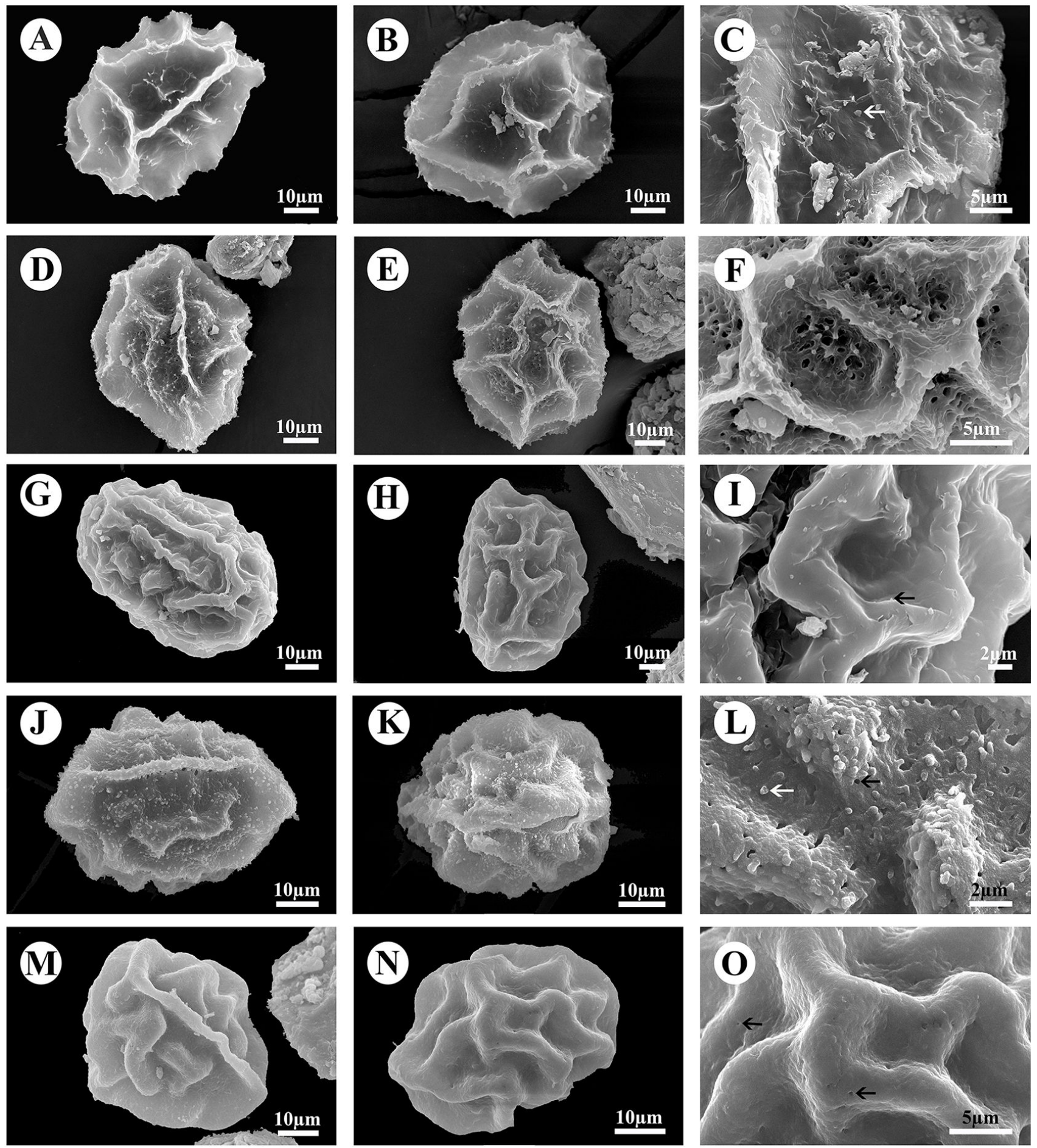

Figura 2. Esporas de Aspleniaceae, Cystopteridaceae y Dryopteridaceae. A-C Asplenium fibrillosum. A) Vista proximal, lesura monolete. B) Vista distal, ornamentación reticulada a lofada. C) Detalle de vista proximal, presencia de escasas microespinas (flecha). D-F Asplenium monanthes. D) Vista proximal, lesura monolete. E) Vista distal, perisporio plegado. F) Perisporio con pliegues con forma de crestas, microequinas y foveolos. G-I Asplenium praemorsum. G) Vista proximal, lesura monolete. H) Vista distal, ornamentación plegada con patrón hamulado. I) Detalle de vista proximal, perisporio plegado con escasas microespinas (flecha). J-L Cystopteris fragilis. J) Vista proximal, lesura monolete. K) Vista distal, perisporio con pliegues. L) Detalle de vista distal, ornamentación escábrida con gránulos y perforaciones (flechas). M-O Dryopteris cinnamomea. M) Vista proximal, lesura monolete. N) Vista distal, ornamentación hamulada. O) Detalle de vista, perisporio con pocas perforaciones (flechas). 
Asplenium monanthes L.- Esporas monoletes con persiporio evidente. VP: ovoidal a elipsoidal con margen irregular determinado por los pliegues del perisporio. VE: planoconvexa. O: Regular en toda la superficie, el perisporio presenta pliegues con forma de crestas de $5.9 \mu \mathrm{m}$ de longitud que forman un patrón retículo-hamulado con lumen de 5 a $10 \mu \mathrm{m}$. Las crestas presentan microespinas y en el lumen se observan foveolos de $1 \mu \mathrm{m}$ (Figura 2D-F). Ejemplares: Pérez-Jiménez 34, 55, 58, 78, 91, 124, 136, MEXU folio:1465157. Para Asplenium monanthes el diámetro polar de las esporas en este trabajo se reportó de 25-30 $\mu \mathrm{m}$ y el ecuatorial de 25-50 $\mu \mathrm{m}$ (Tabla 1), considerablemente menores a 39-45 $\mu \mathrm{m}$ y $53-60 \mu \mathrm{m}$, diámetros reportados por Tryon \& Lugardon (1991) y Gómez-Noguez et al. (2013), posiblemente esta variación en tamaño se deba a que esta especie pertenece a un complejo (Dyer et al. 2012)

Asplenium praemorsum Sw.- Esporas monoletes con perisporio evidente, hasta $8 \mu \mathrm{m}$ de ancho. VP: elipsoidal con márgenes irregulares por el pliegue del perisporio. VE: planoconvexa a reniforme. O: regular en toda su superficie, se observa la superficie del perisporio plegado, con un patrón hamulado, los muri de 2 a $4 \mu \mathrm{m}$ de alto con microespinas escasas. (Figura 2G-I). Ejemplares: PérezJiménez 12, 15, 32, 48, 61, 73, 74, 86, 89, 108, 121, 143, 153, MEXU folio:1465156. Murillo \& Bless (1978) indican que el diámetro ecuatorial para Asplenium praemorsum es de $37-45 \mu \mathrm{m}$ y el polar de $18-25 \mu \mathrm{m}$, mientras que en este estudio son ligeramente más grandes de 47.5-67.5 $\mu \mathrm{m}$ y 25-47.5 $\mu \mathrm{m}$ (Tabla 1$)$.

Cystopteridaceae Shmakov. Cystopteris fragilis (L.) Bernh.- Esporas monoletes con perisporio evidente. VP: elipsoidal, en ocasiones de margen irregular por los pliegues del perisporio. VE: planoconvexas. O: regular en toda la superficie, los pliegues del perisporio irregulares, superficie escábrida con gránulos y perforaciones (Figura 2J-L). Ejemplar: Pérez-Jiménez 87, MEXU depositado sin folio. El diámetro y ornamentación de las esporas de este taxón, concuerdan con lo señalado por Arreguín-Sánchez et al. (1996) y corresponden con el morfo-tipo "Cave" propuesto por Parks et al. (2000), pero no coinciden con el tamaño y la descripción de GómezNoguez et al. (2013) y Murillo \& Bless (1978). Esta variación puede deberse a que Cystopteris fragilis pertenece a un complejo (Murillo \& Bless 1978).

Dyopteridaceae Herter. Dryopteris cinnamomea (Cav.) C. Chr.- Esporas monoletes. VP: elipsoidales con márgenes irregulares por los pliegues del perisporio. VE: elipsoidales a reniformes. O: regular en toda la superficie, los pliegues del perisporio de 5 a $7 \mu \mathrm{m}$ de alto, forman un patrón hamulado. Se observa el perisporio con pocas perforaciones, (Figura 2M-O). Ejemplares: Pérez-Jiménez 16, 30, 35, 43, 64, 70, 88, 126, 145, 154, MEXU folio: 1465163. Hernández-Hernández et al. (2009) señalan para Dryopteris cinnamomea que el diámetro polar es de 27-36 $\mu \mathrm{m}$ y el ecuatorial de 31-43 $\mu \mathrm{m}$. En contraposición el tamaño de los especímenes estudiados fue ligeramente mayor con un diámetro polar de 22.0-30.0 $\mu \mathrm{m}$ y el ecuatorial de 36.0-48.0 $\mu \mathrm{m}$ (Tabla 1).

Ophioglossaceae Martinov. Ophioglossum engelmannii Prantl.- Esporas triletes. VP: esferoidal a subtriangular con lados convexos y ángulos redondeados. VE: semiesférica, convexo-plano a cóncavo. O: reticulada, con lumen de $1 \mathrm{a}$ $2.5 \mu \mathrm{m}$, irregular, más grande en la zona ecuatorial, menor en cara proximal, muri escábrido; de 0.5 a $1.1 \mu \mathrm{m}$ de grosor, más ancho en zona ecuatorial (Figura 3A-C, $\underline{8 B}$ ). Ejemplar: Pérez-Jiménez 150, MEXU depositado sin folio.

Polypodiaceae Bercht. \& J. Presl. Phlebodium pseudoaureum (Cav.) Lellinger.- Esporas monoletes. VP: elipsoidal a ovalada. VE: plano-convexa a planocóncava (reniforme). O: verrugada, las verrugas son más pequeñas en la cara proximal que en vista distal y están ausentes en la zona cercana a la lesura, estos elementos son hialinos (Figura 3D-F). Ejemplares: Pérez-Jiménez 1, 4, 21 , 53, 139, 152, MEXU folio: 1465152.

Pleopeltis thyssanolepis (A. Braun ex Klotzsch) E.G.Andrews \& Windham.- Esporas monoletes. VP: ovalada a elipsoidal. VE: plano-convexa a cóncavoconvexa (reniforme). O: regular en toda la superficie. Verrugada con elementos hialinos redondeados y dispersos irregularmente en la superficie (orbículas o cuerpos esferoidales); el exosporio hialino presenta un grosor de $2 \mu \mathrm{m}$ (Figuras 3G-I, $\underline{8 \mathrm{H}}$ ). Ejemplares: Pérez-Jiménez 3, 9, 28, 38, 60, 83, 92,115, 151, MEXU folio: 1465162.

Polypodium subpetiolatum Hook. in Bentham.- Esporas monoletes con perisporio evidente. VP: elipsoidal. VE: plano-convexa. O: Verrugada a fosulada, las verrugas son más grandes en la zona ecuatorial y distal. El exosporio tiene un grosor de aproximadamente $3 \mu \mathrm{m}$ (Figura 3J-L). Ejemplares: Pérez-Jiménez 29, 129, 159, MEXU folio: 1465164.

Nota: en ocasiones las verrugas se disponen entre ellas de manera tal que forman surcos dando la apariencia de un patrón fosulado. Para esta especie Arreguín-Sánchez et al. (1996) citan que el diámetro polar es de 30-32 $\mu \mathrm{m}$ y el ecuatorial es de $46-53 \mu \mathrm{m}$. No obstante, los diámetros observados en este estudio, fueron ligeramente mayores, el polar es de $25.0-44.0 \mu \mathrm{m}$ y el ecuatorial de $37.0-59.0 \mu \mathrm{m}$ (Tabla 1). 

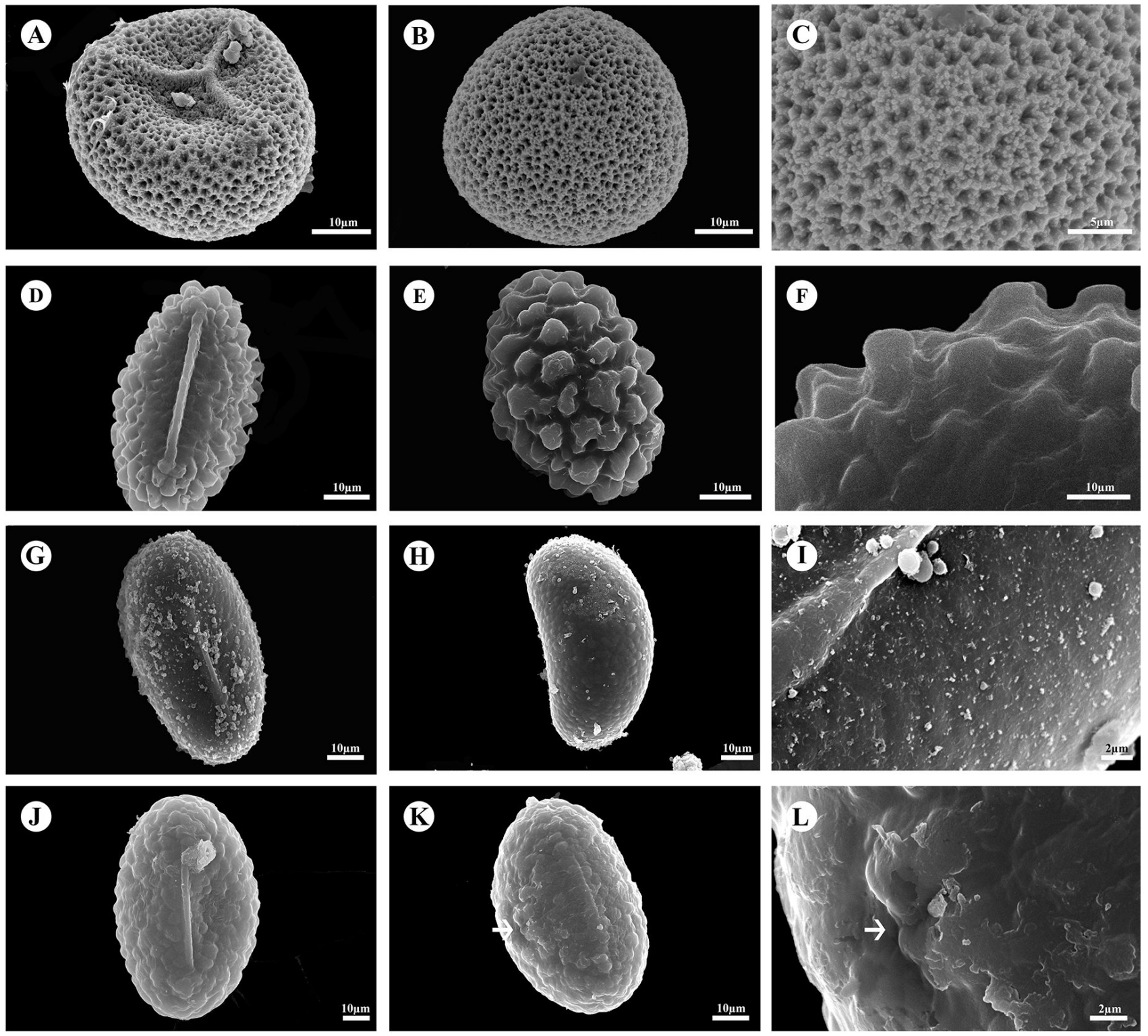

Figura 3. Esporas de Ophlioglossaceae y Polypodiaceae. A-C Ophioglossum engelmannii. A) Vista proximal, lesura trilete. B) Vista distal, ornamentación reticulada. C) Detalle de vista distal ornamentación muri escábrida. D-F Phlebodium pseudoaureum. D) Vista proximal, lesura monolete. E) Vista distal, ornamentación verrugada. F) detalle de las verrugas. G-I Pleopeltis thyssanolepis. G) Vista proximal, lesura monolete. H) Vista distal, ornamentación verrugada, verrugas aisladas. I) Detalle de vista proximal, orbículas (cuerpos esferoidales). J-L Polypodium subpetiolatum. J) Vista proximal, lesura monolete. K) Vista distal, ornamentación verrugada con surcos (flecha). L) Vista proximal, detalle de las verrugas y surco (flecha).

Pteridaceae E. D. M. Kirchn. Adiantum capillus-veneris L.Esporas triletes, con perisporio muy delgado. VP: subtriangular a esferoidal, lados convexos y ángulos redondeados. VE: esferoidal, convexa en ambas caras a convexo-cónico O: regular en toda la superficie, escábrido con escasos cuerpos esferoidales (Figura 4A-C). Ejemplares: Pérez-Jiménez 132, 135 y 146, MEXU folio: 1465151.
Nota: la forma y ornamentación fue descrita por Tryon \& Lugardon (1991) y Murtaza et al. (2006), aquí se aportan los diámetros del eje polar y ecuatorial.

Astrolepis sinuata (Lag. ex Sw.) D.M. Benham \& Windham. Esporas triletes. VP: Subtriangular a esferoidal. VE: semiesférica, convexo-cónico a convexo-plano. O: cara distal reticulada, heterobrocada con lúmen de 7 a $20 \mu \mathrm{m}, \mathrm{y}$ 

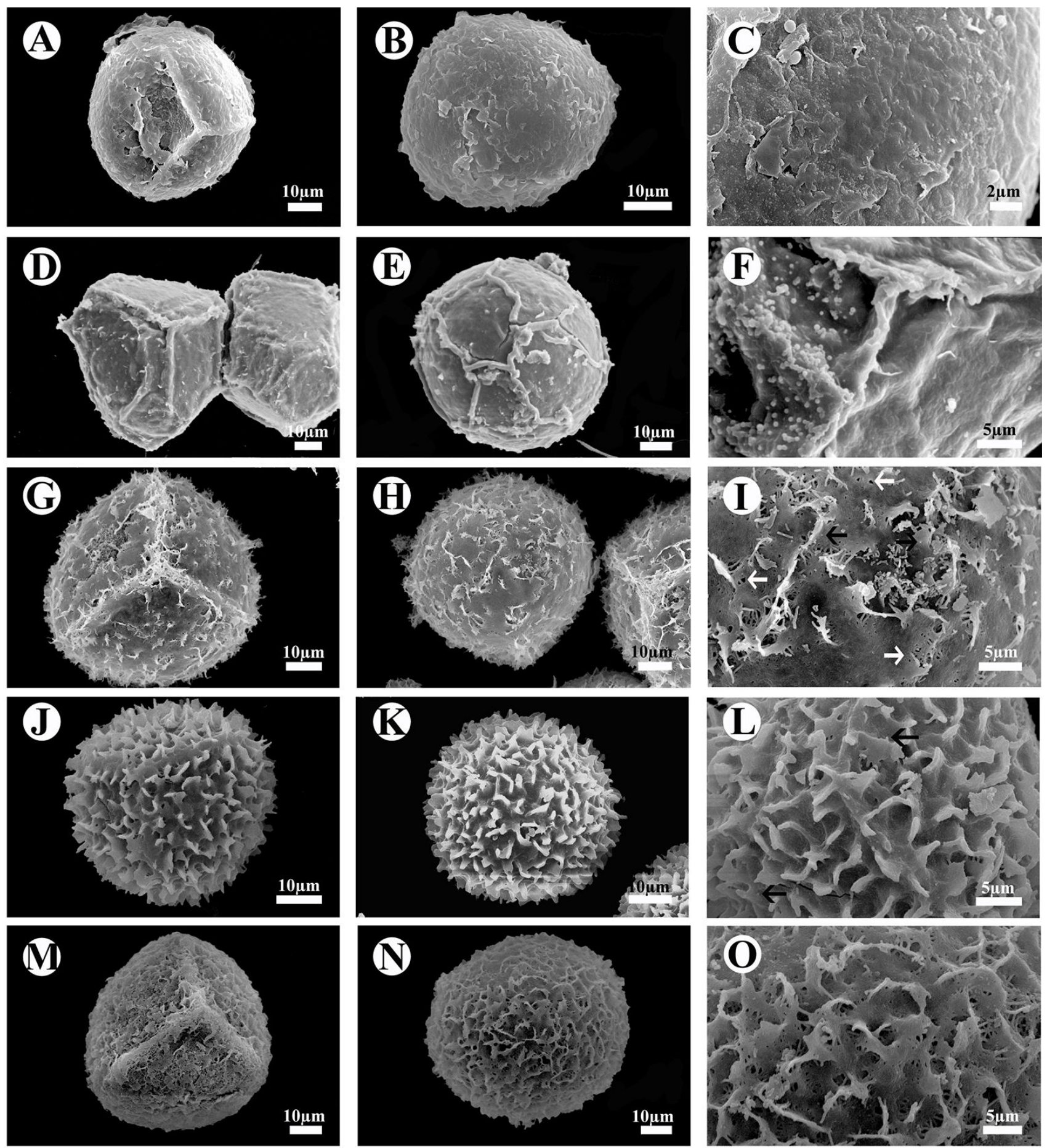

Figura 4. Esporas de Pteridaceae I. A-C Adiantum capillus-veneris. A) Vista proximal, lesura trilete. B) Vista distal, ornamentación escábrida con escasas orbículas. C) Vista distal, detalle de las orbículas. D-F Astrolepis sinuata. D) Vista proximal, lesura trilete, ornamentación psilada con algunos pliegues y orbículas. E) Vista distal, ornamentación reticulada heterobrocada y muri. F) Detalle de las orbículas. G-I Bommeria pedata. G) Vista proximal, lesura trilete. H) Vista distal, ornamentación rugulada con crestas dispersas con perforaciones. I) Vista distal, detalle de las crestas con cordones parcialmente fusionados formando redes (flechas). J-L Cheilanthes farinosa. J) Vista proximal, lesura trilete. K) Vista distal, ornamentación con crestas cortas distribuidas uniformemente. L) Detalle de las espinas (flecha negra) y perforaciones (flecha blanca). M-O Gaga decomposita. M) Vista proximal, lesura trilete. N) Vista distal, ornamentación crestada con patrón hamulado. O) Detalle de las crestas con cordones parcialmente fusionados formando redes en la base. 
muri de 1 a $2.5 \mu \mathrm{m}$, cara proximal psilada con algunos pliegues. Sobre la superficie se observan cuerpos esferoidales (Figura 4D-F). Ejemplares: Pérez-Jiménez 140, 157, MEXU folio: 1465159.

Nota: Para esta especie la descripción presentada aquí en cuanto al tamaño, forma y ornamentación, no coincide con lo citado por Morbelli \& Ponce (1997). Cabe mencionar que esta especie pertenece al complejo de Adiantum sinuata propuesto por Benham \& Windham (1992), quienes mencionan que en las especies de este complejo existe gran variación en la micromorfología de las esporas e, inclusive, en la lesura, ya que un mismo ejemplar puede exhibir esporas aletes, monoletes y triletes.

Bommeria pedata (Sw.) E. Fourn.- Esporas triletes. VP: esferoidal. VE: esferoidal a convexo-cónica. O: mayormente rugulada a veces formando un patrón reticulado, las rúgulas con muri de 1 a $5 \mu \mathrm{m}$; se observan crestas dispersas, la superficie de la base de las crestas parece estar formada por cordones parcialmente fusionados formando redes. Los espacios entre las redes se asemejan a perforaciones (Figura 4G-I). Ejemplares: Pérez-Jiménez 27, 39, 80, 109, 120, MEXU folio: 1465166.

Nota: Para esta epecie Vivero-Barón et al. (2012) reportaron un diámetro polar de 46.4-54.8 $\mu \mathrm{m}$ y el ecuatorial de 27.4-35.8 $\mu \mathrm{m}$, mientras que en esta investigación son mayores con 52.5-58.0 $\mu \mathrm{m}$ y 53.0$78.0 \mu \mathrm{m}$ (Tabla 1).

Cheilanthes farinosa (Forssk.) Kaulf.- Esporas triletes. VP: subtrialgular a esferoidal. VE: convexa-cónica a esferoidal $\mathrm{O}$ : crestas cortas distribuidas uniformemente sobre la superficie, con escasas espinas y perforaciones (Figura 4J-L). Ejemplares: Pérez-Jiménez 49, 52, 125, MEXU folio: 1465167.

Gaga decomposita (M. Martens \& Galeotti) F. W. Li \& Windham.- Esporas triletes con perisporio evidente. VP: esferoidal a subtriangular. VE: esferoidal a convexacónica. O: crestada, las crestas son menores a $5 \mu \mathrm{m}$ de altura formando un patrón hamulado a incompletamente reticulado que posee lúmenes de 2 a $5 \mu \mathrm{m}$. Se observan perforaciones en la base de las crestas, resultado de la fusión parcial de cordones (Figura 4M-O y $\underline{8 \mathrm{C}}$ ). Ejemplares: Pérez-Jiménez 25, 46, 148, 149, MEXU folio: 1465154.

Nota: Las esporas estudiadas en este trabajo son idénticas a las descritas por Nayar (1963) y de diámetros ligeramente mayores a los mencionados por Arreguín-Sánchez et al. (1996). Sin embargo, difieren por completo con las descripciones de Srivastava \& Uniyal (2014).

Gaga kaulfussii (Kunze) F. W. Li \& Windham.- Esporas triletes con perisporio evidente. VP: subtriangular con lados convexos. VE: esferoidal a convexa-cónica. O: escábrida con algunas perforaciones. El perisporio está formado por cordones parcialmente fusionados, formando redes (Figura 5A-C). Ejemplares: Pérez-Jiménez 19, 33, 56, 77, 82, 107, 110, 127, 131, MEXU depositado sin folio.

Nota: Knobloch (1969) describe la forma, ornamentación y el diámetro del eje ecuatorial, en este estudio se describe tambien el diámetro del eje polar.

Gaga membranacea (Davenp.) F.W. Li \& Windham.Esporas triletes. VP: subtriangular con lados convexos a esferoidal. VE: semiesférica a convexo-cónica. O: crestada, las crestas formadas por pliegues a veces formando un patrón reticulado (Figuras 5D-F, $\underline{\text { 8D) }}$. Ejemplares: PérezJiménez 147, 149, MEXU despositado sin folio.

Myriopteris allosuroides (Mett.) Grusz \& Windham.Esporas triletes. VP: subtriangular con lados convexos a esferoidal. VE: esferoidal. O: escábrida con pocas perforaciones. El perisporio presenta una superficie

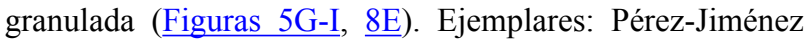
26, 137, MEXU depositado sin folio.

Myriopteris aurea (Poir.) Grusz \& Windham.- Esporas triletes con perisporio evidente. VP: subtriangular con lados convexos a esferoidal. VE: hemiesférico-plano a hemiesférico-convexo. O: escábrida con pocas perforaciones y con pliegues menores a $1 \mu \mathrm{m}$. Algunas esporas presentan superficie foveolada, e inclusive pueden observarse esporas puntuadas a perforadas (Figura 5J-L). Ejemplares: Pérez-Jiménez 2, 6, 24, 36, 37, 41, 54, 66, 72, 79, 85, 96, 101, 104, 111, MEXU folio: 1465165.

Myriopteris myriophylla (Desv.) Sm.- Esporas triletes con perisporio evidente. VP: esferoidal. VE: esferoidal. O: escábrida, foveolas (Figura 5M-O). Ejemplares: PérezJiménez 5, 8, 17, 31, 44, 67, 75, 81, 93, 98, 106, 113,117, MEXU folio: 1465172.

Pellaea cordifolia (Sessé \& Moç.) A.R. Sm.- Esporas triletes. VP: subtriangular con lados rectos a cóncavos y ápices redondeados, a esferoidal. VE: convexo-cónico a esferoidal. O: rugulada con pliegues y crestas (Figura 6A-C y $\underline{8 \mathrm{~F}}$ ). Ejemplares: Pérez-Jiménez 10, 18, 23, 45, 63, 95, 102, 118, MEXU folio: 1465170.

Pellaea ternifolia (Cav.) Link.- Esporas triletes con perisporio evidente. VP: subtriangular con lados cóncavos y ápices obtusos, a esferoidal. VE: convexo-cónica a esferoidal. O: rugulada, cada rúgula mide aproximadamente $2 \mu \mathrm{m}$ de ancho (Figura 6D-F). Ejemplares: Pérez-Jiménez 7 , 11, 14, 22, 50, 76, 97, 100, 105, 119, 156, MEXU folio: 1465169. 

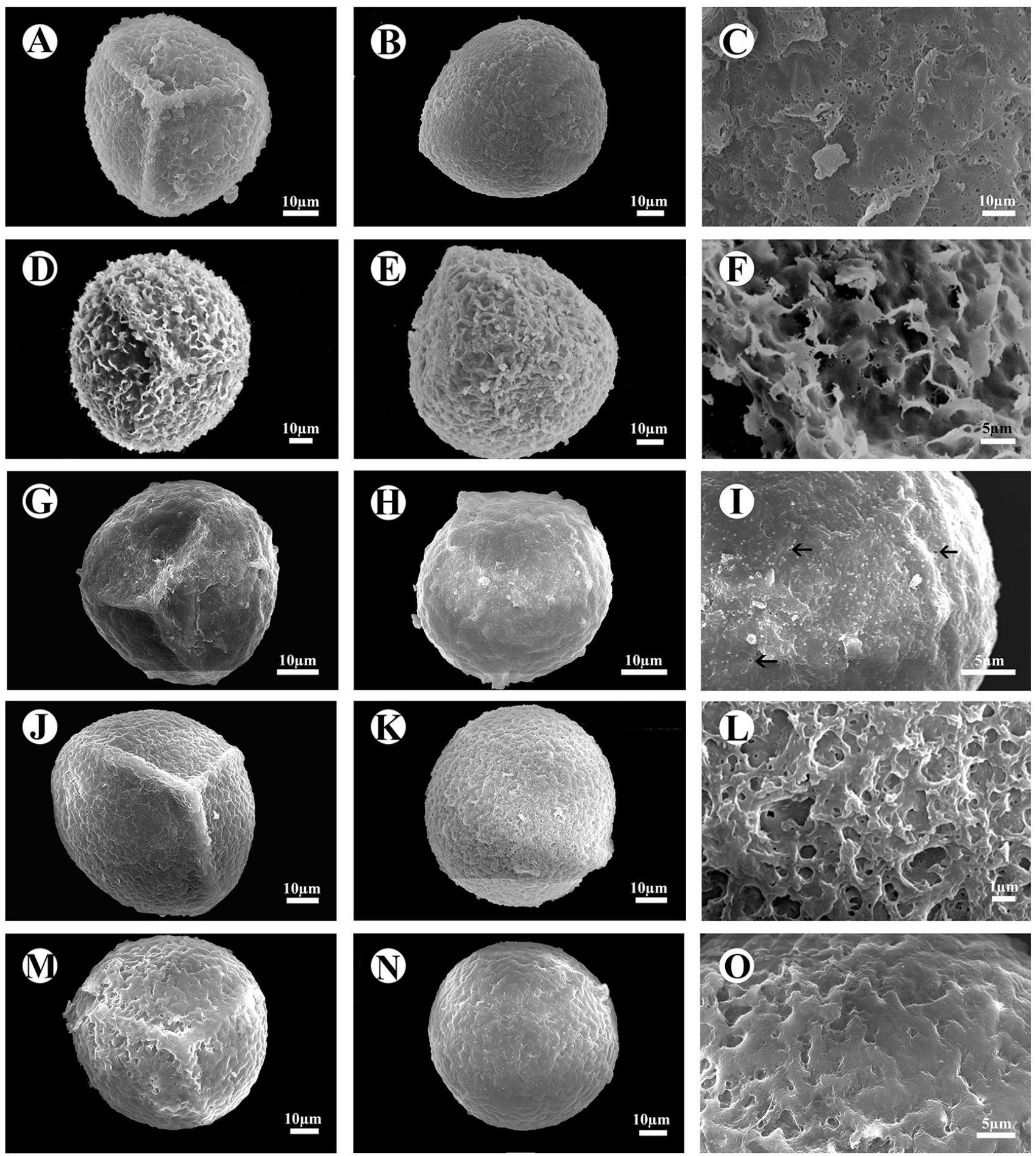

Figura 5. Esporas de Pteridaceae II. A-C Gaga kaulfussii. A) Vista proximal, lesura trilete. B) Vista distal, ornamentación escábrida con algunas perforaciones. C) Detalle de las redes de cordones. D-F Gaga membranacea. D) Vista proximal, lesura trilete. E) Vista distal, ornamentación crestada. F) Detalle de crestas. G-I Myriopteris allosuroides. G) Vista proximal, lesura trilete. H) Vista distal, ornamentación escábrida. I) Detalle de la ornamentación escábrida con perforaciones escasas (flecha). J-L Myriopteris aurea. J) Vista proximal, lesura trilete. K) Vista distal, escábrida con pocas perforaciones. L) Se observan pliegues menores a una $\mu \mathrm{m}$, superficie foveolada M-O Myriopteris myriophylla. M) Vista proximal, lesura trilete. N) Vista distal, ornamentación escábrida. O) Detalle de vista proximal, escábrida con foveolos. 
[

Pérez-Jiménez et al. / Botanical Sciences 98(3): 517-532. 2020
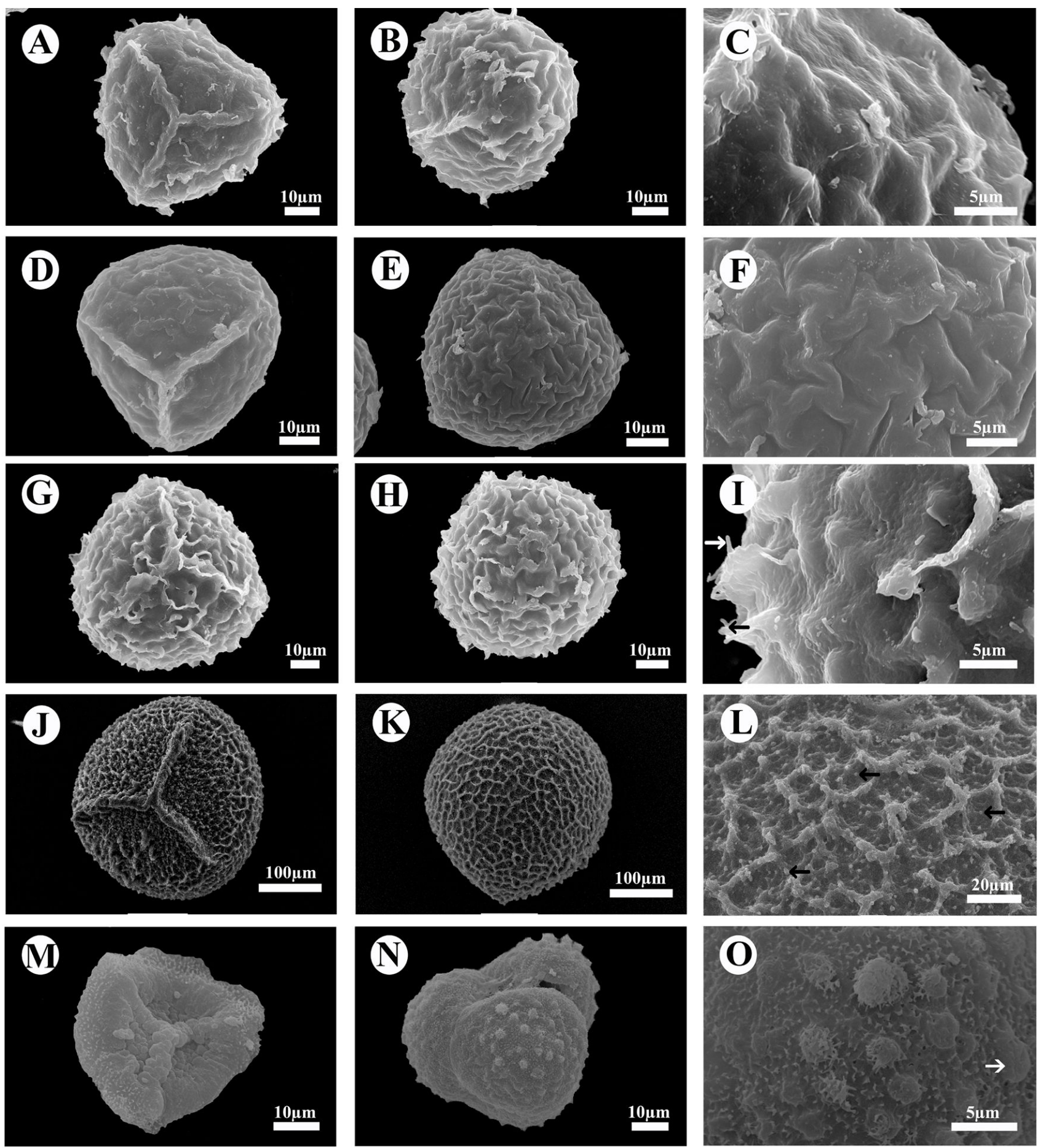

Figura 6. Esporas de Pteridaceae III, Selaginellaceae I. A-C Pellaea cordifolia. A) Vista proximal, lesura trilete. B) Vista distal, ornamentación rugulada con pliegues y crestas escasas. C) Detalle de los pliegues en vista proximal. D-F Pellaea ternifolia. D) Vista proximal, lesura trilete. E) Vista distal, ornamentación rugulada. F) Detalle de las rúgulas, vista distal. G-I Pellaea villosa. G) Vista proximal, lesura trilete. H) Vista distal, ornamentación rugulada. I) Detalle de las rúgulas con báculas (fecha) dispuestas aleatoriamente. J-L Selaginella pallescens, megasporas. J) Vista proximal, lesura trilete, ornamentación cerca de la lesura papilada y crestada a estriada-reticulada hacia la región del cíngulo. K) Vista distal, ornamentación reticulada heterobrocada a estriada-reticulada. L) Detalle de la retícula con orbículas (flecha), vista distal. M-O Selaginella pallescens, microsporas. M) Vista proximal, lesura trilete, verrugada a gemada cerca de la lesura. N) Tétrada inmadura con gemas y microespinas. O) Detalle de las clavas (flecha) y de las microespinas, vista distal. 
Pellaea villosa (Windham) Windham \& Yatsk.- Esporas triletes. VP: subtriangular, ápices obtusos y lados convexos, a esferoidal. VE: convexo-cónica. O: rugulada, rúgulas con báculas en el ápice dispuestas aleatoriamente (Figuras 6G-I, $\underline{8 \mathrm{G}}$ ). Ejemplares: Pérez-Jiménez 51, 130, MEXU folio: 1465168 .

Selaginellaceae Willk. Selaginella pallescens (C. Presl) Spring in Martius.- Megasporas triletes con cíngulo ecuatorial. VP: subtriangular con ápice obtuso y lados convexos, a esferoidal. VE: convexo-cónica. O: distalmente reticulada heterobrocada a estriada-reticulada, con lumen de 10 a $20 \mu \mathrm{m}$ con perforaciones y cuerpos esferoidales. Proximalmente heterogénea: papilada cerca de la lesura, crestada a estriada-reticulada hacia el cíngulo ecuatorial (Figura 6J-L).

Microsporas triletes en mónadas, diadas y tétradas. VP: esferoidal. VE: plano-convexa a cóncavo-convexa. $\mathrm{O}$ : distalmente clavada (clavas de hasta $6 \mu \mathrm{m}$ de largo) y microcristada, con microespinas en toda la superficie incluyendo los ápices de las clavas, cuando maduras. Proximalmente verrugada a gemada cerca de la lesura, escábrida en esta región y presencia de microespinas en la (Figura 6M-O). Ejemplares: Pérez-Jiménez 13, 20, 47, 57,
59, 65, 68, 71, 94, 99, 112, 123, 144, MEXU folio: 1465160 .

Nota: Las microsporas maduras poseen clavas sin microespinas, mientras que las inmaduras presentan gemas crestadas. Tryon \& Lugardon (1991) reportan una ornamentación papilada-granulada en la cara polar, similares a los que aquí se presentan. Sin embargo, existe una ligera variación en el tamaño: Erdtman \& Sorsa (1971) citan microesporas de $32 \times 54 \mu \mathrm{m}$, Arreguín et al. (2001) de 28-30 × 30-38 $\mu \mathrm{m}$, y Gómez-Noguez et al. (2013) de $22 \times$ $30 \mu \mathrm{m}$, mientras que en el presente el tamaño es de $18 \times$ $24 \mu \mathrm{m}$ (Tabla 1). Estos datos ofrecen información acerca de la variación en el tamaño de las esporas para esta especie. En cuanto a la ornamentación, es similar a lo observado por otros autores, pero las medidas de los elementos como son: espinas, papilas, granulas, entre otras, varían entre las poblaciones y grado de maduración de las esporas, por ello es importante seguir realizando estudios palinológicos, para incrementar esta información.

Selaginella sartorii Hieron.- Megasporas triletes. VP: esferoidal. O: reticulada heterobrocada con muri de 11 a $16 \mu \mathrm{m}$ de ancho, lumen de 50 a $100 \mu \mathrm{m}$, más estrecho cercano a la lesura (Figura 7A-C).
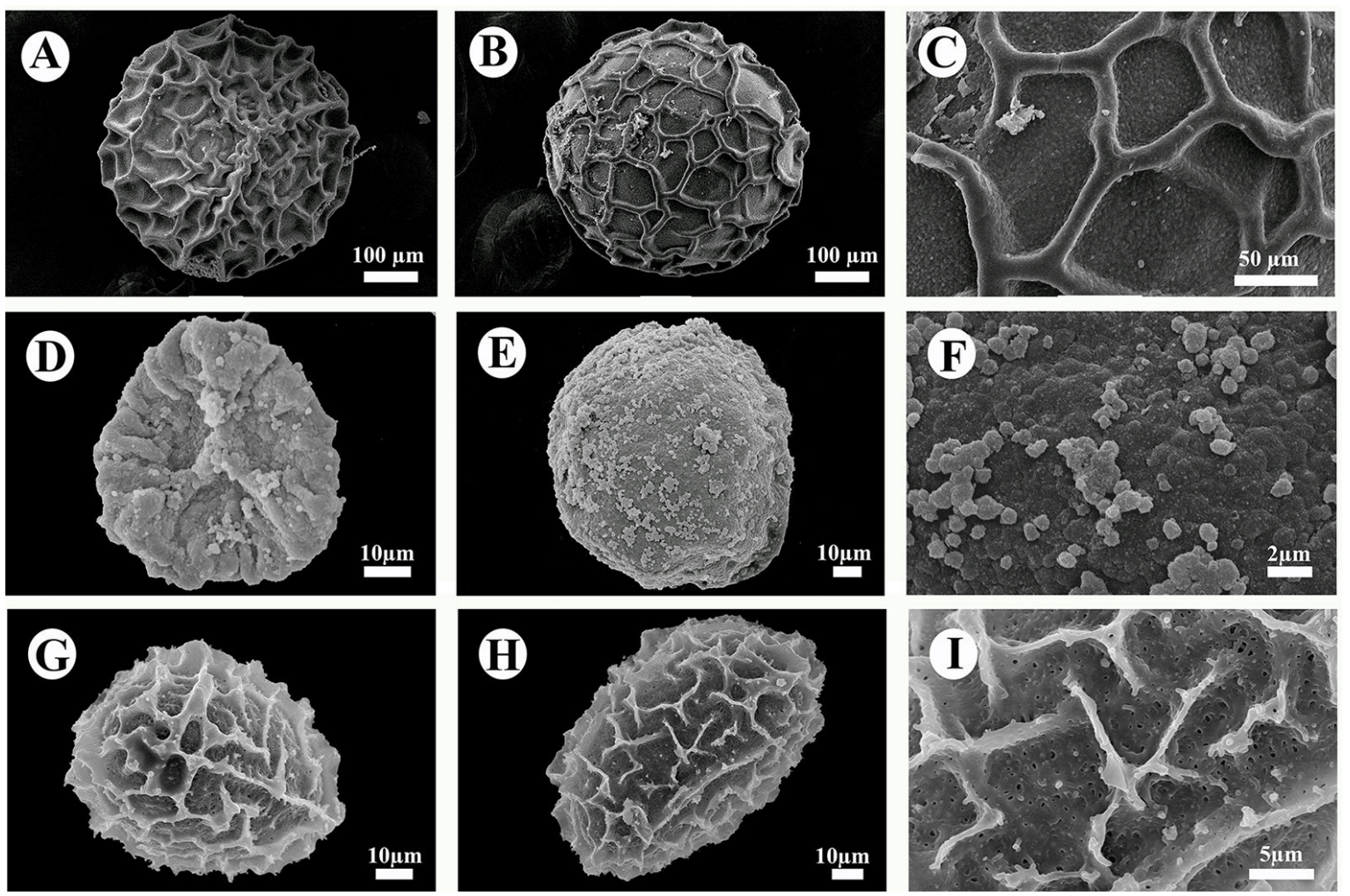

Figura 7. Esporas de Selaginellaceae II y Woodsiaceae. A-C Selaginella sartorii, megasporas. A) Vista proximal, lesura trilete. B) Vista distal, ornamentación reticulada heterobrocada. C) Detalle de vista distal, muri. D-F Selaginella sartorii, microsporas. D) Vista proximal, lesura trilete. E) Vista distal, ornamentación verrucosa-escábrida con orbículas o cuerpos esferoidales. F) Detalle de orbículas, vista distal. G-I Woodsia mollis. G) Vista proximal, lesura monolete. H) Vista distal, perisporio plegado, patrón hamulado. I) El lumen presenta escasas microespinas y abundantes perforaciones. 
Microsporas triletes. VP: subtriangular a esférica. VE: plano-convexa, elipsoidal a esférica. O: verrucosa escábrida con cuerpos esferoidales (Figuras 7D-F, $\underline{8 \mathrm{I}}$ ). Ejemplares: Pérez-Jiménez 114, 155, MEXU folio: 1465161.

Woodsiaceae Herter. Woodsia mollis (Kaulf.) J. Sm.Esporas monoletes con perisporio evidente. VP: elipsoidal. VE: plano-covexa a cóncavo-convexa. O: el perisporio presenta pliegues que forman un patrón reticulado a hamulado. El lumen del retículo presenta gránulos escasos, y abundantes perforaciones. (Figura 7G-I). Ejemplares: Pérez-Jiménez 62, 84, 90, 103, 116, 128, 142, 158. 160, MEXU folio: 1465153.

Nota: Vivero-Barón et al. (2012) indican para Woodsia mollis un diámetro polar de 27.4-35.8 $\mu \mathrm{m}$ y ecuatorial de 46.4-54.8 $\mu \mathrm{m}$, en este trabajo las medidas son de 32.5$47.5 \mu \mathrm{m}$ y de $55.0-70.0 \mu \mathrm{m}$, significativamente más grandes (Tabla 1).

\section{Discusión}

En este trabajo, se describe la micromorfología de las esporas de 25 especies de helechos y licofitas presentes en la ZNP de la REPSA. Para Gaga decomposita, Gaga membranacea, Miryopteris allosuroides, Pellaea cordifolia, Pellaea villosa, Pleopeltis thyssanolepis, Ophioglossum engelmanni y Selaginella sartorii, no existían reportes previos acerca de la micromorfología de sus esporas. En este trabajo se presentan microfotografías en MEB y MF, con la finalidad de evidenciar características novedosas para estas especies. La palinología de las dieciséis especies restantes ya fue descrita completa o parcialmente en investigaciones previas. Específicamente para Asplenium fibrillosum, Adiantum capillus-veneris y Gaga kaulfussii ya existían aportes palinológicos, en este estudio se complementó la información detallada de la micromorfología de las esporas.

En este trabajo se observó que existe una evidente variación en el tamaño de las esporas de los especímenes estudiados con respecto a lo observado por otros autores. Sin embargo, consideramos que el tamaño de las esporas es un carácter de importancia entre los individuos de la misma especie (Barrington et al. 1986, Moran 2004) y la variación de este rasgo puede estar relacionada con la poliploidía (número cromosómico), una característica muy común en los helechos (Brown 1960, Wagner 1974, Barrignton et al. 1986, Moran 2004), o también puede ser consecuencia de las estrategias ecológicas en este tipo de plantas: esporas más grandes tienen una capacidad de dispersión menor y una mayor reserva de nutrientes (Venable 1992). También es necesario considerar que muchas de estas especies forman complejos.
A

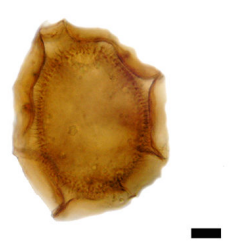

D

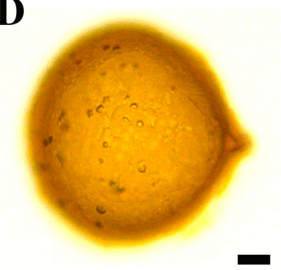

G

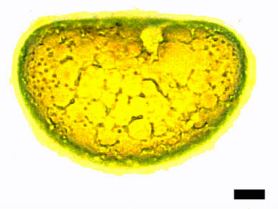

B

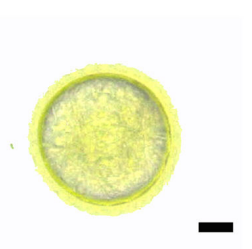

C

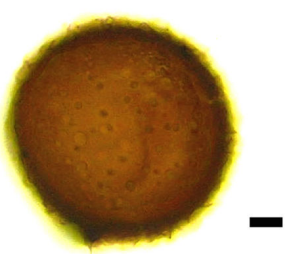

E

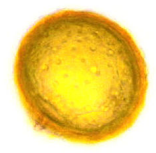

F

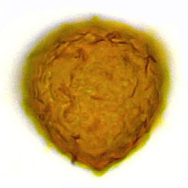

I

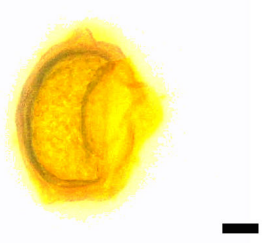

Figura 8. Esporas de las especies descritas por primera vez en vista ecuatorial con MF. A) Asplenium fribrilosum. B) Ophioglossum engelmanni. C) Gaga decomposita. D) Gaga membranacea. E) Myriopteris allosuroides. F) Pellaea cordifolia. G) Pellaea villosa. H) Pleopeltis thyssanolepis. I) Selaginella sartorii, microspora. (barra $=10 \mu \mathrm{m}$ ). 
El conocimiento de la palinología de las especies que habitan en la ZNP de la REPSA reviste importancia, porque conocer la morfología, tamaño y ornamentación de las esporas, puede ayudar en la delimitación taxonómica de las especies y explicar diferentes procesos biológicos de los helechos y licofitas. Desde el punto de vista ecológico conocer la dispersión a corta o larga distancia de las esporas, puede ayudar a comprender los patrones de distribución de estas plantas (Gómez-Noguez et al. 2017). Asimismo, conocer la palinología de las especies de una zona, puede ayudar a conocer e identificar las especies que conforman los bancos de esporas, el estudio de estos bancos es imporntante ya que estos constituyen un reservorio de diversidad genética y pueden contribuir a la regeneración de ecosistemas alterados por actividades antropogénicas (Dyer 1994). Finalmente, conocer la morfología de las esporas provee una referencia para investigaciones paleobotánicas (Gonçalves de Freitas et al. 2015).

\section{Agradecimientos}

Los autores agradecen el apoyo de la Universidad Nacional Autónoma de México mediante el proyecto PAPIME: PE207116. A la SEREPSA por las facilidades brindadas durante la recolección del material biológico. A Aniceto C. Mendoza Ruiz por su ayuda en la determinación de las especies. Y a los revisores anónimos que ayudaron a mejorar sustancialmente este manuscrito con sus atinadas observaciones.

\section{Literatura citada}

Arreguín ML, Palacios-Chávez R, Quiroz DL. 2001. Pteridoflora ilustrada del estado de Querétaro, México. DF México: Escuela Nacional de Ciencias Biológicas IPN. 470 pp. ISBN: 968-7377-40-2.

Arreguín-Sánchez MDL, Fernández-Nava R, PalacioChávez R, Quiroz-García DLQ. 1996. Morfología de las esporas de pteridófitas isospóreas del estado de Querétaro, México. Polibotánica 2: 10-60.

Arreguín-Sánchez ML, Aguirre-Claverán R. 1990. Morfología de las esporas isospóreas triletes de Pteridófitas de Nuevo León, México. Palynologica et Paleobotanica 2: 5-62.

Arreguín-Sánchez ML, Fernández-Nava R, PalaciosChávez R, Quiroz-García DL. 1996. Morfología de las esporas de pteridófitas isospóreas del estado de Querétaro, México. Polibotánica 2: 10-60.

Arreguín-Sánchez ML. 1985. Morfología de las esporas de la subfamilia Asplenoideae del Valle de México. In: Montúfar A, eds. Estudios Palinológicos y Paleoetnobotánicos. México, DF: Instituto Nacional de Antropología e Historia, 7-22.
Barrington DS, Paris CA, Ranker A. 1986. Systematic inferences from spore and stomate size in the ferns. American Fern Journal 76: 149-159. DOI: https:// doi.org/10.2307/1547723

Benham DM, Windham MD. 1992. Generic affinities of the star-scaled cloak ferns. American Fern Journal 82: 47-58. DOI: https://doi.org/10.2307/1547377

Brown C A. 1960. What is the role of spores in Fern taxonomy? American Fern Journal 50: 6-14. DOI: https://doi.org/10.2307/1545238

Cano-Santana Z, Domínguez-Álvarez L, Moyers- Arévalo R, Velázquez- López R, García-Jiménez C. 2009. La Reserva Ecológica Del Pedregal De San Ángel: Estudios Ecosistémicos. [CD]. Memorias in extenso CoFC1507e-25 VII Congreso sobre Áreas Naturales Protegidas de México. San Luis Potosí, jul. 13-17. Organizado por SEMARNAT, Comisión de Áreas Naturales Protegidas y el Gobierno del Estado de San Luis Potosí.

Chao YS, Huang YM. 2018. Spore morphology and its systematic implication in Pteris (Pteridaceae). PLOS ONE 13: e0207712. DOI: https://doi.org/10.1371/jour nal.pone. 0207712

Contreras-Duarte AR, Bogotá-Ángel RG, Jiménez-Bulla LC. 2006. Atlas de las esporas de pteridófitos de Chipaque (Cundinamarca, Colombia). Caldasia 28: 327-357. DOI: http://dx.doi.org/10.15446/caldasia

Devi S, Nayar BK, Knobloch IW. 1971. Spore Morphology of some American Species of Cheilanthes and Notholaena. Grana 11: 27-35. DOI: https://doi.org/ $\underline{10.1080 / 00173137109427408}$

Dyer AF. 1994. Natural soil spore banks - Can they be used to retrive lost ferns? Biodiversity and Conservation 3: 160-175. DOI: https://doi.org/10.1007/BF02291886

Dyer RJ, Savolainen V, Schneider H. 2012. Apomixis and reticulate evolution in the Asplenium monanthes fern complex. Annals of Botany 110: 1515-1529. DOI: https:// doi.org/10.1093/aob/mcs202

Erdtman G, Sorsa P. 1971. Pollen and Spore Morphology / Plant Taxonomy: Pteridophyta. Estocolmo: Almqvist \& Wiksell.

Freitas, AG de, Carvalho, MA, Toledo, MB, Mendonça, CBF, Gonçalves-Esteves V. 2015. Bryophyte and Pteridophyte spores and other palynomorphs in quaternary marine sediments from Campos Basin, Southeastern Brazil: Core BU-91-GL-05. Acta Botanica Brasilica 29:161-174. DOI: https://doi.org/10.1590/ $\underline{\text { 0102-33062014abb3734 }}$

Gastony GJ. 1979. Spore morphology in the Cyatheaceae. III. The genus Trichipteris. American Journal of Botany 66: 1238-1260. https://doi.org/10.1002/j.1537-2197.19 79.tb06342.x 
Gómez-Noguez F, Pérez-García B, Mendoza-Ruíz A, Orozco-Segovia A. 2013. Flora Palinológica de los Helechos y Licofitas de Río Malila, Hidalgo, México. Botanical Sciences 91: 135-154. DOI: https://doi.org/ $\underline{10.17129 / \text { botsci.410 }}$

Gómez-Noguez F, Pérez-García B, Mendoza-Ruiz A, Orozco-Segovia A. 2017. Fern and lycopod spores rain in a cloud forest of Hidalgo, Mexico. Aerobiologia 33: 23-35. https://doi.org/10.1007/s10453-016-9447-1

Hagenah DJ. 1961. Spore studies in the genus Cystopteris I. The distribution of Cystopteris with non-spiny spores in North America. Rhodora 63: 181-193.

Hernández-Hernández V, Terrazas T, Delgadillo MC. 2009. The Dryopteris patula complex (Dryopteridaceae) in Mexico: morphometric analyses. Botanical Sciences $\mathbf{8 5}$ : 103-112. DOI: http://dx.doi.org/10.17129/botsci.2308

Hill SR. 1977. Spore morphology of Anemia subgenus Coptophyllum. American Fern Journal 67: 11-17. DOI: https://doi.org/10.2307/1546195

Hill SR. 1979. Spore morphology of Anemia subgenus Anemia. American Fern Journal 69: 71-79. DOI: https:// doi.org/10.2307/1546381

Knobloch IW, Spink GC, Fulfs JS. 1971. Preliminary scanning electron microscope observations on the relief of the spore wall of some Cheilanthoid ferns. Grana 11: 23-26. DOI: https://doi.org/10.1080/00173137109427 $\underline{407}$

Knobloch IW. 1969. The spore pattern in some species of Cheilanthes. American Journal of Botany 56: 646-653. DOI: https://doi.org/10.1002/j.1537-2197.1969.tb07581. $\underline{\mathrm{x}}$

Korall P, Taylor WA. 2006. Megaspore morphology in the Selaginellaceae in a phylogenetic context: A study of the megaspore surface and wall structure using scanning electron microscopy. Grana 45: 22-60. DOI: https:// doi.org/10.1080/00173130500520453

Kremp GOW, Kawasaki T. 1972. The spores of the pteridophytes. Tokio: Hirokawa Publishing Company.

Kremp GOW. 1965. Morphologic Encyclopedia of Palynology, An International Collection of Definitions and Illustrations of Spores and Pollen. Tucson: The University of Arizona Press, ISBN-13: 978-0816500604

Labiak PH, Rouhan G, Sundue M. 2010. Phylogeny and taxonomy of Leucotrichum (Polypodiaceae): A new genus of grammitid ferns from the Neotropics. Taxon 59: 911-921. DOI: https://doi.org/10.1002/tax.593019

Lehtonen S, Tuomisto H, Rouhan G, Christenhusz MJM. 2010. Phylogenetics and classification of the pantropical fern family Lindsaeaceae. Botanical Journal of the Linnean Society 163: 305-359. DOI: https://doi.org/ 10.1111/j.1095-8339.2010.01063.x

Lellinger D, Taylor CA. 1997. Classification of Spore Ornamentation in the Pteridophyta. In: Johns RJ, ed.
Holttum Memorial Volume: published to commemorate the centenary of the birth of Professor R. E. Holttum. UK: Royal Botanical Gardens, Kew. ISBN-13: 9781900347174

Lellinger DB. 2002. A Modern Multilingual Glossary for Taxonomic Pteridology. Pteridologia 3. Washington, DC: American Fern Society, ISBN-13: 978-0933500020

Lloyd RM. 1981. The perispore in Polypodium and related genera (Polypodiaceae) Canadian Journal of Botany 59: 175-189. DOI: https://doi.org/10.1139/b81-028

Lot A, Chiang F. 1986. Manual de herbario: Administración y manejo de colecciones y preparación de ejemplares botánicos. México: Consejo Nacional de la Flora de México,. ISBN: 9686144005

Mickel JT, Beitel JM. 1988. Pteridophyte Flora de Oaxaca, México. New York USA: New York Botanical Garden. ISBN-13: 978-0893273231

Mickel JT, Smith AR. 2004. The Pteridophytes of Mexico. New York: Memories of the New York Botanical Garden. NY press. ISBN-13: 978-0893274887

Moran RC. 2004. A Natural History of Ferns. Portland: Timber Press, ISBN-13: 978-1604690620.

Moran RC, Hanks JG, Rouhan G. 2007. Spore morphology in relation to phylogeny in the fern genus Elaphoglossum (Dryopteridaceae). International Journal of Plant Sciences 168: 905-929. DOI: https://doi.org/10.1086/ $\underline{518269}$

Moran RC, Riba R.1995. "Psilotaceae a Salviniaceae". In: Davidse E, Sousa M, Knapp S, eds. Flora Mesoamericana. Vol. 1. Psilotaceae a Slavinaceae. México, DF: Universidad Nacional Autónoma de México, Mussouri Botanical Garden, The Natural History Museum (London). 300. ISBN: 9683647006

Morbelli MA, Ponce MM. 1997. Palynological Study of Cheilanthes and Astrolepis (Pteridaceae) Species from Northwestern Argentina. American Fern Journal 87: 51-65. DOI: $\underline{\text { https://doi.org/10.2307/1547536 }}$

Muñiz-Díaz de León ME, Pérez-García B, MárquezGuzmán J, Mendoza-Ruiz A. 2008. Developmental gametophyte morphology of seven species of Thelypteris subg. Cyclosorus (Thelypteridaceae). Micron 39: 1351-1362. DOI: https://doi.org/10.1016/j.micron.2008. $\underline{02.001}$

Murillo MT, Bless MJ. 1978. Spores of recent Colombian pteridophyta. II. Monolete spores. Review of Palaeobotany and Palynology 25: 319-365. DOI: https:// doi.org/10.1016/0034-6667(78)90019-2

Murtaza G, Asghar R, Majid SA, Waheed A, Mirza SN. 2006. Anatomical and palynological studies on some Filicales from Neelum valley, Muzaffarabad, Azad Kashmir. Pakistan Journal of Botany. 38: 921.

Nayar BK, Devi S. 1966. Spore morphology of the Pteridaceae I. the Pteridoid ferns. Grana 6: 476-502. DOI: https://doi.org/10.1080/00173136609430036 
Nayar BK. 1963. The morphology of some species of Cheilanthes. Journal of the Linnean Society 58: 449-460. DOI: https://doi.org/10.1111/j.1095-8339.1963.tb00914.x Parks JC, Dyer AF, Lindsay S. 2000. Allozyme, spore and frond variation in some Scottish populations of the ferns Cystopteris dickieana and Cystopteris fragilis. Edinburgh Journal of Botany. 57: 83-105. DOI: https:// doi.org/10.1017/s0960428600000068

PPG. 2016. A community-based classification for extant ferns and lycophytes. Journal of Systematics and Evolution. 54: 563-603. DOI: https://doi.org/10.1111/ jse. 12229

Punt W, Hoen PP, Blackmore S, Nilsson S, Le Thomas A. 2007. Glossary of pollen and spore terminology. Review Paleobotany and Palynology 143: 1-81. DOI: https:// doi.org/10.1016/j.revpalbo.2006.06.008

Ramírez-Valencia V, Sanín D, Pardo-Trujillo A. 2013. Análisis morfológico de las esporas de Serpocaulon (Polypodiaceae) de la Cordillera Central de Colombia. Caldasia 35: 177-197.

Ramírez-Valencia V, Sanín D. 2017. Spores of Serpocaulon (Polypodiaceae): morphometric and phylogenetic analyses. Grana 56: 187-203. DOI: https://doi.org/ $\underline{10.1080 / 00173134.2016 .1184307}$

Rouhan G, Hanks JG, McClelland D, Moran RC. 2007. Preliminary phylogenetic analysis of the fern genus Lomariopsis (Lomariopsidaceae). Brittona 59: 115-128. DOI: https://doi.org/10.1663/0007-196x(2007)59[115:pp aotf]2.0.co;2

Schneider H, Pryer KM. 2002. Structure and function of spores in the aquatic heterosporous fern family Marsileaceae. International Journal of Plant Sciences 163: 485-505. DOI: https://doi.org/10.1086/340736

\footnotetext{
Associate editor: Alejandra Vasco

Contribución de los autores: JCPJ: colecta, identificación del material de respaldo de la investigación y de esporas, y técnicas metodológicas para el montaje de éstas para MF y MEB. FJES: colecta de especímenes para elaborar los ejemplares de herbario y escritura del artículo. KJD: adquisición de microfotografías por MEB y elaboración de las figuras. FGN: descripción de las esporas. MEMDL: responsable del proyecto, infraestructura, insumos, colecta y escritura del artículo. Todos los autores revisaron el artículo.
}

Srivastava R, Uniyal PL. 2014. In-vitro developmental studies of Cheilanthes farinosa (Forssk.) Kaulf. (Pteridaceae). Telopea 1787-91. DOI: http://dx.doi.org/ $\underline{10.7751 / \text { telopea20147554 }}$

Tryon AF, Lugardon B. 1991. Spores of the Pteridophyta: Surface, Wall Structure and Diversity Based on Electron Microscope Studies. New York NY: Springer-Verlag. ISBN: 978-1-4613-8993-4: DOI: https://doi.org/10.100 7/978-1-4613-8991-0

Tryon RM, Tryon AF. 1982. Additional taxonomic and nomenclatural notes on ferns. Rhodora. 84: 125-130.

Valiente-Banuet A, De Luna GE. 1990. Una lista florística actualizada para la Reserva del Pedregal de San Ángel, México D.F. Acta Botanica Mexicana 9: 13-30. DOI: https://doi.org/10.21829/abm9.1990.588

Venable DL. 1992. Size-number trade-offs and the variation of seed size with plant resource status. The American Naturalist 140: 287-304. DOI: https://doi.org/10.108 $\underline{6 / 285413}$

Vivero-Barón J, Arreguín-Sánchez ML, Quiroz-García DL, Fernández- Nava R, Acosta-Castellanos S. 2012. Seguimiento del Ciclo de Vida de Woodsia mollis (kaulf.) J. Sm. (Athyriaceae-Pteridophyta) en Tres Sustratos Naturales. Polibotánica 33: 75-83.

Wagner WH. 1974. Structure of spores in relation to fern phylogeny. Annals of the Missouri Botanical Garden 61: 332-353. DOI: https://doi.org/10.2307/2395061

Wood CC. 1973. Spore variation in Thelypteridaceae. In: Jeremy AC, Crabbe JA, Thomas BA. eds. The Phylogeny and Classification of the Ferns, Academic Press, London, 191-201. ISBN: 9780123850508 